\title{
Self-Consistent Pushing and Cranking Corrections to the Meson Fields of the Chiral Quark-Loop Soliton
}

\author{
M. SChLEIF ${ }^{1}$, R. WÜNSCH${ }^{1}$ AND T. MEISSNER ${ }^{2}$ \\ ${ }^{1}$ Institut für Kern- und Hadronenphysik, Forschungszentrum Rossendorf e. V., \\ Postfach 5101 19, D-01314 Dresden, Germany \\ 2 Department of Physics, Carnegie-Mellon-University, Pittsburgh, PA 15213, \\ USA
}

\begin{abstract}
We study translational and spin-isospin symmetry restoration for the two-flavor chiral quark-loop soliton. Instead of a static soliton at rest we consider a boosted and rotating hedgehog soliton. Corrected classical meson fields are obtained by minimizing a corrected energy functional which has been derived by semi-classical methods ('variation after projection').

We evaluate corrected meson fields in the region $300 \mathrm{MeV} \leq M \leq$ $600 \mathrm{MeV}$ of constituent quark masses $M$ and compare them with the uncorrected fields. We study the effect of the corrections on various expectation values of nuclear observables such as the root-mean square radius, the axial-vector coupling constant, magnetic moments and the delta-nucleon mass splitting.
\end{abstract}




\section{Introduction}

Chiral soliton models have turned out to be a fruitful approach to the structure of light baryons. We consider the soliton of the two-flavor chiral quark-loop model, which is equivalent to the semi-bosonized Nambu \& Jona-Lasinio (NJL) model, with hedgehog meson fields restricted to the chiral circle (for a detailed review cf. ref. [1, 2]). Mesons are treated as mean fields (zero boson loop) whereas the polarization of the Dirac sea is fully taken into account. In order to obtain a system with baryon number $B=1$ one has to add $N_{\mathrm{c}}=3$ valence quarks occupying the lowest single-particle level with positive energy. Static, self-consistent meanfield solitons are obtained by minimizing the effective energy. For a detailed description of procedure, formalism and notation we refer the reader to ref. [3].

As it is well known the mean-field hedgehog soliton breaks both the translational and rotational symmetry. The soliton is not an eigenstate of the total linear $(\vec{P})$ and angular $(\vec{J})$ momentum and of the isospin operator $(\vec{T})$. As a result, the expectation value $\left\langle\vec{P}^{2}\right\rangle$ does not vanish, and $\left\langle\vec{J}^{2}\right\rangle$ and $\left\langle\vec{T}^{2}\right\rangle$ do not have the values appropriate for a nucleon or a $\Delta$ isobar. The energy functional, which defines the solitonic field configuration, is contaminated by spurious contributions. There are various method to exclude the spurious energies and to determine the energy of a state with definite quantum numbers. In non-relativistic many-particle physics the spurious energies can be obtained within a certain approximation using Peierls-Yoccoz [4] or Peierls-Thouless [5] projection techniques for the linear and angular momenta. Unfortunately those methods require the definition of a Fock state which is very involved if dealing with the polarized and regularized Dirac sea. In the case of the Skyrme model it has turned out that the consideration of RPA fluctuations around the mean-field solution allows a treatment of the corresponding eigenmodes [6, 7] analogously to the case of two-dimensional soliton models [8]. Recently this approach has also been used to calculate the quantum corrections to the effective soliton energy in the chiral quark-loop model within some approximations [9].

In the present paper we follow a simplified approach and rely on the semiclassical pushing and cranking approximations [10]. Hereby one is considering a soliton with the appropriate quantum numbers by pushing the static hedgehog with the velocity $V$ and rotating its isospin coordinates with the angular velocity $\Omega$ where $V$ and $\Omega$ are regarded as Lagrange multipliers. Due to the hedgehog symmetry the angular momentum of the soliton is fixed to $\vec{J}=-\vec{T}$ and one has automatically $\left\langle\vec{J}^{2}\right\rangle=\left\langle\vec{T}^{2}\right\rangle$. All the studies which have been done so far performed the projection after the variation, which means that first the mean field configurations are calculated by minimizing the static soliton energy and afterwards the rotational and translational correction terms are added. It is the aim of this paper to investigate if and how the properties of the chiral quark-loop model change if the more accurate procedure of a variation after projection is carried out. In this case one has to minimize a soliton energy which includes the 
correction term. The resulting corrected meson fields respond to the collective translation and rotation of the soliton and deviate from the static ones. The inertial parameters correspond to those values which minimize the corrected energy functional (self-consistent cranking). Expectation values of quark and meson observables are also modified.

In sect. 2, we define the static hedgehog soliton and introduce a corrected energy functional for a soliton with definite momentum and isospin. The field configuration which minimizes the corrected energy functional is determined in sect. 3. Particular attention is drawn to the asymptotic behavior of the meson profile. In sect. 4 we investigate the effect of the corrections on solitonic expectation values. Conclusions are drawn in sect. 5 .

\section{Static mean-field and energy corrections}

In mean-field approximation, the $\mathrm{SU}(2)$ NJL lagrangian describes $u$ and $d$ quarks interacting with classical meson fields $\sigma$ and $\hat{\vec{\pi}}$. Restricted to time-independent spherical hedgehog configurations $(\sigma(t, \vec{r})=\sigma(r), \vec{\pi}(t, \vec{r})=\pi(r) \hat{\vec{r}})$ and to the chiral circle $\left(\sigma(r)^{2}+\pi(r)^{2}=M^{2}\right)$ with unit vector $\hat{\vec{r}} \equiv \vec{r} / r, r=|\vec{r}|$, the meson fields are uniquely determined by the constituent quark mass $M$ and the profile function $\Theta(r) \equiv \arctan \frac{\pi(r)}{\sigma(r)}$. The mean-field energy

$$
E_{\mathrm{mf}}[\Theta]=E^{\mathrm{m}}[\Theta]+E^{\mathrm{q}}[\Theta]
$$

is a functional of $\Theta$ and consists of a purely mesonic part

$$
E^{\mathrm{m}}[\Theta]=\frac{m_{0} M}{G} 4 \pi \int r^{2} d r[1-\cos \Theta(r)]
$$

and of the quark energy

$$
E^{\mathrm{q}}[\Theta]=-\lim _{T_{\mathcal{E}} \rightarrow \infty} \frac{1}{T_{\mathcal{E}}} \operatorname{Tr} \operatorname{Ln}\left[\partial_{\tau}+h\right]+\lim _{T_{\mathcal{E}} \rightarrow \infty} \frac{1}{T_{\mathcal{E}}} \operatorname{Tr} \operatorname{Ln}\left[\partial_{\tau}+h_{0}\right]+N_{\mathrm{c}} \varepsilon_{\text {val }} \Theta\left(\varepsilon_{\text {val }}\right)
$$

with the single-particle quark hamiltonians

$$
h=-\mathrm{i} \vec{\alpha} \cdot \vec{\nabla}+\beta M e^{\mathrm{i} \gamma_{5} \hat{\vec{\tau}} \cdot \overrightarrow{\vec{r}} \Theta(r)} \quad \text { and } \quad h_{0}=-\mathrm{i} \vec{\alpha} \cdot \vec{\nabla}+\beta M .
$$

Here, $\vec{\alpha} \equiv \beta \vec{\gamma}, \vec{\gamma} \equiv\left(\gamma^{1}, \gamma^{2}, \gamma^{3}\right), \gamma_{5} \equiv \mathrm{i} \gamma^{0} \gamma^{1} \gamma^{2} \gamma^{3} \gamma^{4}$ and $\beta \equiv \gamma^{0}$ are Dirac matrices, $m_{0}$ is an average current quark mass $m_{0}=\left(m_{\mathrm{u}}+m_{\mathrm{d}}\right) / 2$. The Euclidean timecoordinate is denoted by $\tau$, while $\hat{\vec{\tau}}$ denotes the vector of Pauli matrices. The last term in eq. (3) describes the energy of the valence quarks which have been added to ensure a baryon number $B=1$ for the soliton. It vanishes if the meson field is so strong that the energy $\varepsilon_{\text {val }}$ of the valence level is negative. In this case the Dirac sea has already $B=1$. The trace $\operatorname{Tr}$ in eq. (3), which includes 
functional trace with anti-periodic boundary conditions in the Euclidean time interval $\left(-\frac{T_{\mathcal{E}}}{2}, \frac{T_{\mathcal{E}}}{2}\right)$ and matrix trace over Dirac, flavor and color indices, can be expressed by a regularized sum over the eigenvalues of the hamiltonians $h$ and $h_{0}$. For details $c f$. ref. [3].

The classical meson profiles have to minimize the mean-field energy (1)

$$
\frac{\delta E_{\mathrm{mf}}}{\delta \Theta(r)}=0
$$

leading to the equation of motion

$$
\Theta(r)=\arctan \frac{\tilde{P}(r)}{\tilde{S}(r)},
$$

which is an implicit equation since scalar and pseudoscalar quark densities $\tilde{S}(r)$ and $\tilde{P}(r)$ (cf.ref. [3]) are functionals of the meson fields. At large separations from the center of the soliton the meson fields approach to their vacuum values $\sigma(r \rightarrow \infty)=\sigma_{0}=M$ and $\pi(r \rightarrow \infty)=0$, while the difference $\Theta(0)-\Theta(\infty)$ is related to the baryon number of the soliton. In order to obtain a soliton with baryon number $B=1$ we assume

$$
\Theta(r) \longrightarrow\left\{\begin{array}{ll}
-\pi & \text { for } r \rightarrow 0 \\
0 & \text { for } r \rightarrow \infty
\end{array} .\right.
$$

The implicit equation of motion (6) is usually solved iteratively. Starting from a reasonable profile $\Theta^{0}$ one diagonalizes the hamiltonians (4) and gets a set of eigenvalues and eigenfunctions which allow to determine the densities $\tilde{S}$ and $\tilde{P}$. The latter are used on the right side of eq. (6) in order to get an improved profile. This procedure is repeated unless the profile remains unchanged within a certain accuracy. It has been shown 11] that the iteration converges for constituent quark masses $M \gtrsim 330 \mathrm{MeV}$. Mesonic fields which fulfill the equation of motion (6) with quark fields which diagonalize the corresponding quark hamiltonian (4) are called self-consistent solutions of the NJL model in mean-field approximation. They have to be distinguished from a parameterized meson field with a predetermined shape which approximates the self-consistent field and does not fulfill the equation of motion (6) in general.

The soliton as a mean-field solution with hedgehog shape is not an eigenstate of total momentum $\vec{P}$ and isospin operator $\vec{T}$. As a result, the expectation values $\left\langle\vec{P}^{2}\right\rangle$ and $\left\langle\vec{T}^{2}\right\rangle$ do not have the values

$$
\left\langle\vec{P}^{2}\right\rangle=0 \quad \text { and } \quad\left\langle\vec{T}^{2}\right\rangle=T(T+1)
$$

with $T=\frac{1}{2}$ for a nucleon and $T=\frac{3}{2}$ for a $\Delta$ isobar at rest. The mean field distinguishes a definite position (the center) of the soliton and the hedgehog defines 
a privileged direction ( $\tau_{z}$ is maximal in $z$ direction) what obviously contradicts translational and rotational symmetry. The mean-field hedgehog soliton performs spurious motions (oscillations around the center of mass and in isospace). These spurious motions are not only responsible for the deviation of $\left\langle\vec{P}^{2}\right\rangle$ and $\left\langle\vec{T}^{2}\right\rangle$ from the physical values (8) but contribute to most of the expectation values of the soliton.

The energy of the spurious modes can be approximately calculated in the framework of the pushing and cranking approaches often used in non-relativistic many-particle physics [10]. After semi-classical quantization [12] of the rotational degrees of freedom the energy of a soliton with the expectation values (8) is given by 13

$$
E_{\mathrm{corr}}^{T}=E_{\mathrm{mf}}-E_{\text {trans }}^{0}-E_{\mathrm{rot}}^{0}+E_{\mathrm{crank}}^{T}
$$

with the translational zero-point (center-of-mass) energy

$$
E_{\text {trans }}^{0}=\frac{\left\langle\vec{P}^{2}\right\rangle_{\mathrm{hh}}}{2 M^{\text {inert }}}
$$

and its iso-rotational equivalent

$$
E_{\mathrm{rot}}^{0}=\frac{\left\langle\vec{T}^{2}\right\rangle_{\mathrm{hh}}}{2 I}=\frac{9}{8 I} .
$$

The parameters $M^{\text {inert }}$ and $I$ are the Thouless-Valatin inertial parameters of translational and rotational motion of the soliton as a whole. As shown in [13] the inertial mass $M^{\text {inert }}$ coincides with the static mean-field energy (11). The expectation value $\left\langle\vec{P}^{2}\right\rangle_{\text {hh }}$ of the mean-field hedgehog soliton consists of contributions from valence and sea quarks and is a functional of the meson profile. It diverges and therefore has to be regularized. We apply Schwinger's proper-time scheme [14] and relate the cut-off parameter to the constituent quark mass by means of the weak pion decay. For details see refs. [11, 15, 16, 17.

The expectation value of $\left\langle\vec{T}^{2}\right\rangle_{\mathrm{hh}}$ is finite. In contrast to the corresponding value for the linear momentum it is independent of the shape of the profile function and consists of the single-particle expectation values of the 3 valence quarks $\left\langle\vec{T}^{2}\right\rangle_{\mathrm{hh}}=3 \cdot \frac{1}{2}\left(\frac{1}{2}+1\right)=\frac{9}{4}$. The Dirac sea does not contribute to the isospin of the hedgehog soliton. The iso-rotational moment of inertia $I$ is given by a regularized Inglis formula [15] and has to be calculated numerically. A term like (11) has a well known analog in nuclear physics, namely the band-head energy which has to be subtracted from the mean field energy if the cranking procedure is applied to a nucleus with spin different from zero. The last term in eq. (9)

$$
E_{\mathrm{crank}}^{T}=\frac{T(T+1)}{2 I}
$$


is the genuine cranking term and results from the global quantized rotation which was introduced to obtain the correct value for the isospin. Both the rotational corrections (111) and (12) can be considered as the result of a single cranking procedure in the course of which the expectation value $\left\langle\vec{T}^{2}\right\rangle$ has been changed from $\frac{9}{4}$ (resulting from 3 valence quarks) to the physical value (8). The corresponding semi-classically quantized angular velocity is given by

$$
\Omega=-\mathrm{i} \frac{\sqrt{T(T+1)-\left\langle\vec{T}^{2}\right\rangle_{\mathrm{hh}}}}{I}
$$

where the second term in the numerator stems from the rotational zero-point energy of the hedgehog while the factor -i appears because we are working in Euclidean space-time. It is worthwhile to mention that the same correction terms (11) and (12) can be obtained [10] within an approximate projection method using the Kamlah expansion [18]. The various contributions (10 12) to the corrected soliton energy (9) are of different order in $N_{\mathrm{c}}$, namely $E_{\mathrm{mf}} \sim N_{\mathrm{c}}, E_{\text {trans,rot }}^{0} \sim 1$ and $E_{\text {crank }}^{T} \sim 1 / N_{\mathrm{c}}$, even though it will turn out that the cranking term can be numerically of the same order of magnitude as the zero-point energies.

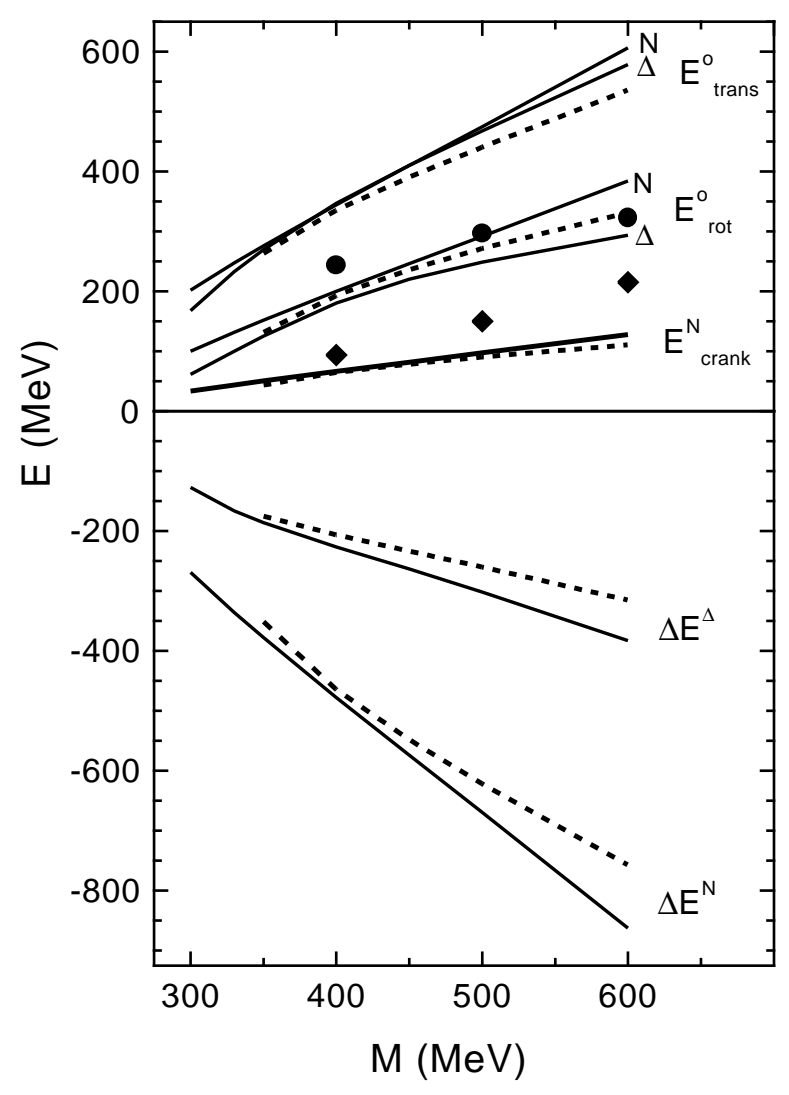

Fig. 1: Total energy corrections $\Delta E^{\mathrm{N}, \Delta}=-E_{\text {trans }}^{0}-E_{\text {rot }}^{0}+E_{\text {crank }}^{\mathrm{N}, \Delta}$ for nucleon and $\Delta$ isobar (lower part) and their components (upper part) calculated for the static soliton profile (broken lines), and for the corrected nucleon $(N)$ and $\Delta$ profiles (full lines) as a function of the constituent quark mass $M$. The cranking energy $E_{\text {crank }}^{\Delta}$ of the $\Delta$ isobar is five times larger than the corresponding energy $E_{\text {crank }}^{\mathrm{N}}$ of the nucleon. The energies of the translational and rotational fluctuations calculated in reference [9] are indicated by $\diamond$ and $\bullet$, respectively.

Fig. 1 illustrates the size of the energy corrections. Each of the terms increases 
approximately linearly with the constituent quark mass $M$. The increase of the corrections is related to the spatial size of the soliton. At larger constituent masses the quarks are stronger bound and the soliton is smaller ( $c f$. fig. 7). Moment of inertia and expectation value $\left\langle\vec{P}^{2}\right\rangle_{\mathrm{hh}}$ behave accordingly. While the moment of inertia decreases the momentum fluctuations increase if the size of the soliton is reduced at larger $M$. Already at small quark masses the corrections amount to a considerable part of the total mean-field soliton energy of roughly $1230 \mathrm{MeV}$. At $M \gtrsim 480 \mathrm{MeV}$ the total energy correction for the nucleon exceeds one half of the soliton energy. For the $\Delta$ isobar the total energy correction is smaller since the zero-point corrections, which have to be subtracted, are partly compensated by the big positive cranking term. Additionally we have indicated the energies of the corresponding mesonic quantum fluctuations calculated in RPA [9]. Comparing them with the semi-classical zero-point energies (10) and (11) one has to take into account that only bound fluctuation have been considered. According to the estimate of ref. [9] unbound fluctuations, which appear in the non-confining N.J model, contribute roughly 50 percent to the translational modes, while unbound rotational modes are negligible.

The considerable size of the energy corrections in comparison to the meanfield energy $E_{\mathrm{mf}}$ conflicts with their perturbative calculation. To improve the approach we shall introduce corrected meson fields which minimize the corrected energy (9) instead of the mean-field energy (11).

\section{Corrected meson profiles}

In sect. 2 we considered the static equation of motion (6) and its solution $\Theta(r)$, in the following called "uncorrected" or "static" profile. The energy corrections 1012) were calculated afterwards using the uncorrected profiles. This approach corresponds to a projection after variation. In the present section we want to perform a variation after projection by minimizing the corrected energy functional (9)

$$
\left.\frac{\delta}{\delta \Theta(r)} E_{\text {corr }}^{T}[\Theta]\right|_{\Theta=\Theta_{\text {corr }}^{T}}=0
$$

from which we obtain "corrected" profiles $\Theta_{\text {corr }}^{T}(r)$. The corrected profiles deviate from the static ones because the inertial parameters $M^{\text {inert }}, I$ and the expectation value $\left\langle\vec{P}^{2}\right\rangle_{\mathrm{hh}}$ depend on the meson profile. Minimizing the corrected energy functional one allows them to acquire a value which corresponds to a lower corrected energy (self-consistent pushing and cranking). Since $E_{\text {corr }}^{T}$ depends explicitly on the isospin quantum number $T$ the corrected profiles are different for nucleons and $\Delta$ isobars.

The lower part of fig. 2 illustrates the difference between the self-consistently determined moments of inertia for nucleons and $\Delta$ isobars and compares them 
with the moment of the static hedgehog soliton. Above $M \approx 380 \mathrm{MeV}$, the moment for the $\Delta$ isobar is roughly $15 \%$ larger than for the nucleon. The harmonic average between both momenta agrees quite well the static moment ( $c f$. sect.4). At smaller constituent quark masses, the mean field has a shallow form and the valence quarks rather weakly bound. In this case the valence quarks are allowed to travel to large distances from the center of the soliton where they are strongly affected by the isospin dependent rotational terms in the corrected effective energy (9). The resulting moments of inertia are large and remarkably different for nucleon and $\Delta$ isobar. The root-mean square (r. m. s.) radius shows a similar behavior (fig. 7). At $M \lesssim 350 \mathrm{MeV}$, the uncorrected mean field is too shallow to produce a bound quark state and a self-consistent solitonic solution of the static equation of motion (6) does not exist. After subtracting the translational zeropoint energy (10) the soliton stabilizes and a self-consistent field configuration exists up to $M \approx 300 \mathrm{MeV}$.

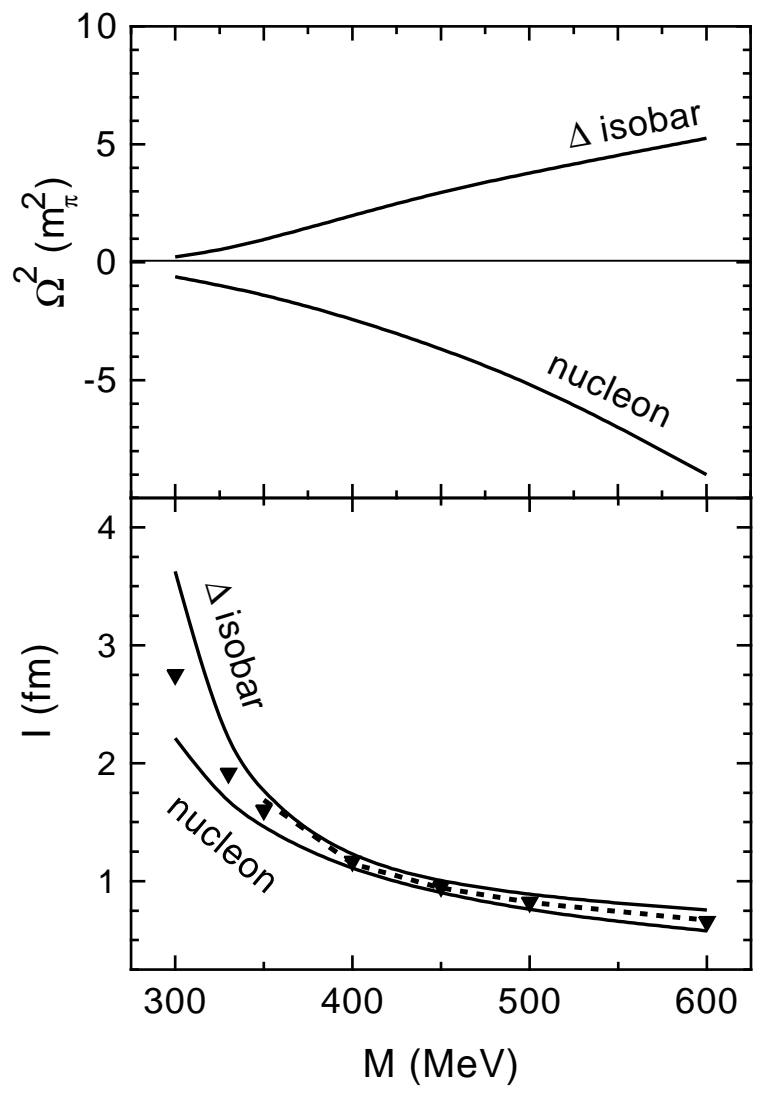

Fig. 2: Lower part:

Self-consistently determined isorotational moments of inertia $I$ (full lines) of nucleon and $\Delta$ isobar in comparison to the static moment of the hedgehog soliton (broken line) as a function of the constituent quark mass $M$. The triangles indicate the harmonic average of the selfconsistent moments of both particles.

Upper part: Lagrange parameter $\Omega^{2}$ according to eq. (13) for nucleon and $\Delta$ isobar as a function of the constituent quark mass.

The upper part of fig. 2 shows the Lagrange parameter $\Omega^{2}$, which is, according to eq. (13), necessary to give the soliton the correct isospin. Since the moment of inertia decreases with increasing constituent quark mass an increasing rotational frequency is needed to reproduce the same isospin. Apart from some minor 
deviations the Lagrange parameters for nucleons and $\Delta$ isobars differ only in the sign. That is why the expectation value $\left\langle\vec{T}^{2}\right\rangle$ lies between the corresponding values for nucleon and isobar. The slight deviations result from the different moments of inertia for either particle.

The corrected equation of motion which follows from condition (14) is much more involved than eq. (6) since the correction terms (10 12) depend on the meson profile. Therefore, we apply a variation method in order to find a profile function $\Theta_{\text {corr }}^{T}(r)$ which minimizes the corrected soliton energy (9). For that aim we represent the profile function by a set of numbers $\Theta_{i},(i=1, \ldots, N)$ and $\tilde{m}_{\pi}$. The $\Theta_{i} \equiv \Theta\left(r_{i}\right)$ are the values of $\Theta(r)$ at $N$ appropriately selected points $r_{1}, \ldots, r_{N}$ (knots) and $\tilde{m}_{\pi}$ characterizes the behavior at $r \rightarrow \infty$. Between the $r_{1}$ and $r_{N}$ the function $\Theta(r)$ will be reproduced by means of a spline interpolation using the $\Theta_{i}$ 's. The asymptotic behavior at small $\left(r<r_{1}\right)$ and large radii $\left(r>r_{N}\right)$ requires particular care. Expanding the profile function of the static soliton in a power series one can show that the quadratic term vanishes at small $r$. The same is true for the corrected profile function where only the slope is modified by the translational zero-point correction. Rotational corrections do not affect the behavior at small radii. Therefore we use a linear ansatz at $r \leq r_{1}$ in agreement with the boundary conditions (7)

$$
\Theta(r) \stackrel{r \rightarrow 0}{\longrightarrow}-\pi+\left(\Theta_{1}+\pi\right) \frac{r}{r_{1}} .
$$

The slope is determined by the variation parameter $\Theta_{1}$.

The behavior at large separations can be determined analytically [17, 19]. Applying the gradient or heat-kernel expansion on the effective action of the chiral soliton in mean-field approximation one can show that the dominating terms agree with the non-linear chiral $\sigma$ model with an additional centrifugal force. The centrifugal term modifies the asymptotic behavior of the meson profile, which is proportional to $\mathrm{e}^{-\mathrm{m}_{\pi} \mathrm{r}}$ at large $r$, in dependence on the rotational frequency $\Omega$. In the plane perpendicular to the rotational axis, the pion mass $m_{\pi}$, which characterizes the exponential descent of the meson field, has to be replaced by a modified pion mass [20, 21, 22

$$
\tilde{m}_{\pi}=\sqrt{m_{\pi}^{2}-\Omega^{2}} .
$$

Since $\Omega^{2}$ is negative for the nucleon and positive for the $\Delta$ isobar ( $c f$.fig. 2) the nucleon is somewhat slimmer (prolate) than the static hedgehog while the isobar is a bit fatter (oblate). Along the cranking axis the soliton is not affected by the rotation. Considering this effect in first order we neglect the deviation from spherical symmetry and introduce a common effective descent parameter $\tilde{m}_{\pi}$ (effective pion mass) for all directions which is considered as an additional variation parameter. The behavior at $r \geq r_{N}$ is then determined by

$$
\Theta(r) \stackrel{r \rightarrow \infty}{\longrightarrow} \Theta_{N}\left(\frac{r_{N}}{r}\right)^{2} \frac{1+\tilde{m}_{\pi} r}{1+\tilde{m}_{\pi} r_{N}} \mathrm{e}^{-\tilde{\mathrm{m}}_{\pi}\left(\mathrm{r}-\mathrm{r}_{N}\right)} .
$$


Restricting ourselves to spherically symmetric solitons we average the effect of the rotation over all directions of the rotational axis. Since only two of the three spatial directions are affected by the rotation we expect roughly $\tilde{m}_{\pi}^{2} \approx m_{\pi}^{2}-\frac{2}{3} \Omega^{2}$ instead of eq. (16). Rotational frequencies $\Omega^{2}>m_{\pi}^{2}$ lead to negative values of $\tilde{m}_{\pi}^{2}$ in eq. (16). The corresponding $\pi$ field oscillates perpendicular to the cranking axis and describes the emission of pions by the soliton. The basis we use for the description of wave functions is defined within a finite box [23] and hence not appropriate for the description of oscillating $\pi$ fields. The effective pion mass resulting from the numerical procedure is close to zero, but real in all cases. In this way the basis prevents the soliton from emitting pions.

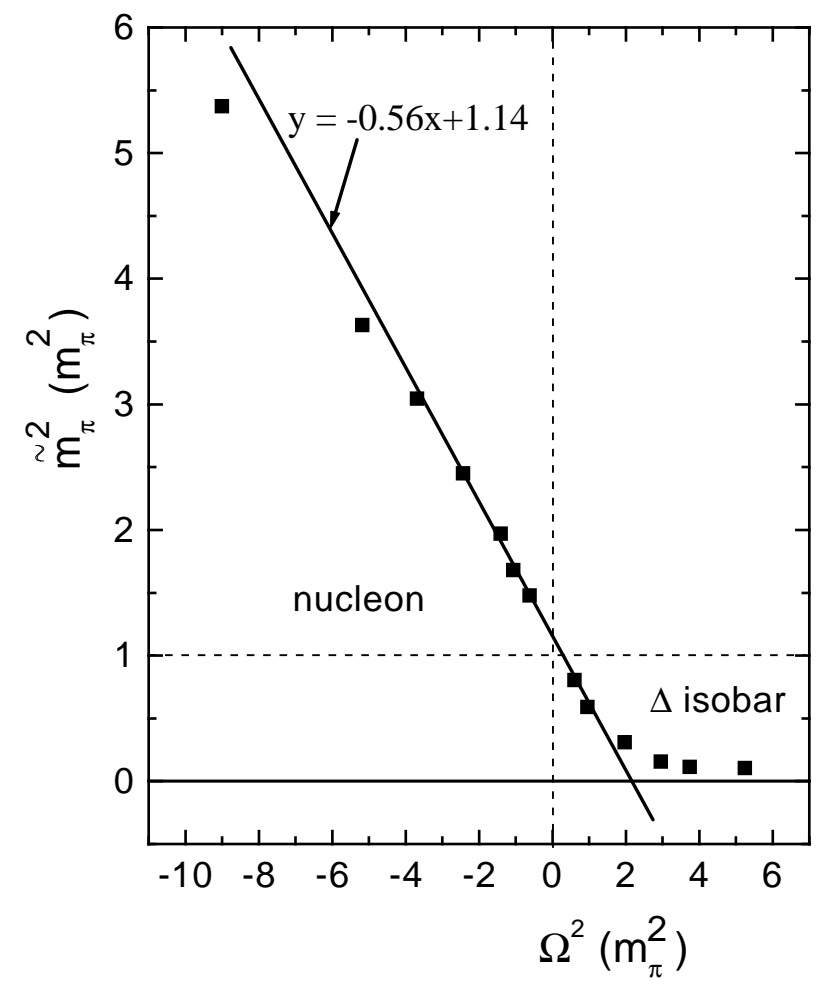

Fig. 3: Square of the effective pion mass $\tilde{m}_{\pi}$, which characterizes the asymptotics of the meson field according to eq. (17), as obtained from minimizing $E_{\text {corr }}^{\mathrm{N}, \Delta}(9)$, as a function of $\Omega^{2}$. Different values $\Omega^{2}$ correspond to different constituent quark masses according to fig. 2 . The line shows a linear fit performed in the region $-6 \leq\left(\Omega / m_{\pi}\right)^{2} \leq 1$.

Fig. 3 displays the numerically determined effective pion masses as a function of $\Omega^{2}$. A fit in the region $-6 m_{\pi}^{2} \leq \Omega^{2} \leq m_{\pi}^{2}$ yields $\tilde{m}_{\pi}^{2} \approx 1.14 m_{\pi}^{2}-0.56 \Omega^{2}$ in fair agreement with our suggestion above. Interpolating to $\Omega^{2}=0$ we get an effective pion mass which deviates from the pion rest mass by 7 percent. A similar deviation (5-10 percent) from the pion rest mass was obtained in a numerical variation without any rotational corrections (only translational corrections or no corrections at all). This demonstrates the accuracy of our procedure. The obvious deviation from the straight line at larger positive values of $\Omega^{2}$ in fig. 3 reveals the limitation to descending pion field in our numerical calculation.

Representing the profile function $\Theta(r)$ by the set of parameters $\Theta_{i},(i=$ 
$1, \ldots, N)$ and $\tilde{m}_{\pi}$ the effective energies (1) and (9) are ordinary functions of $N+1$ variables which can be minimized by standard methods. We tested the method by minimizing the static mean-field energy where the self-consistent profile $\Theta(r)$ can alternatively be determined in the traditional manner by solving the equation of motion (5) iteratively. Here we found that 7 knots are sufficient for an accurate reproduction of the meson profile. Moreover, the optimal position of the knots was determined. Details can be found in ref. [24]. Since the corrected profiles do not substantially deviate from the uncorrected ones we used the same knots for the representation of $\Theta_{\text {corr }}^{T}(r)$.

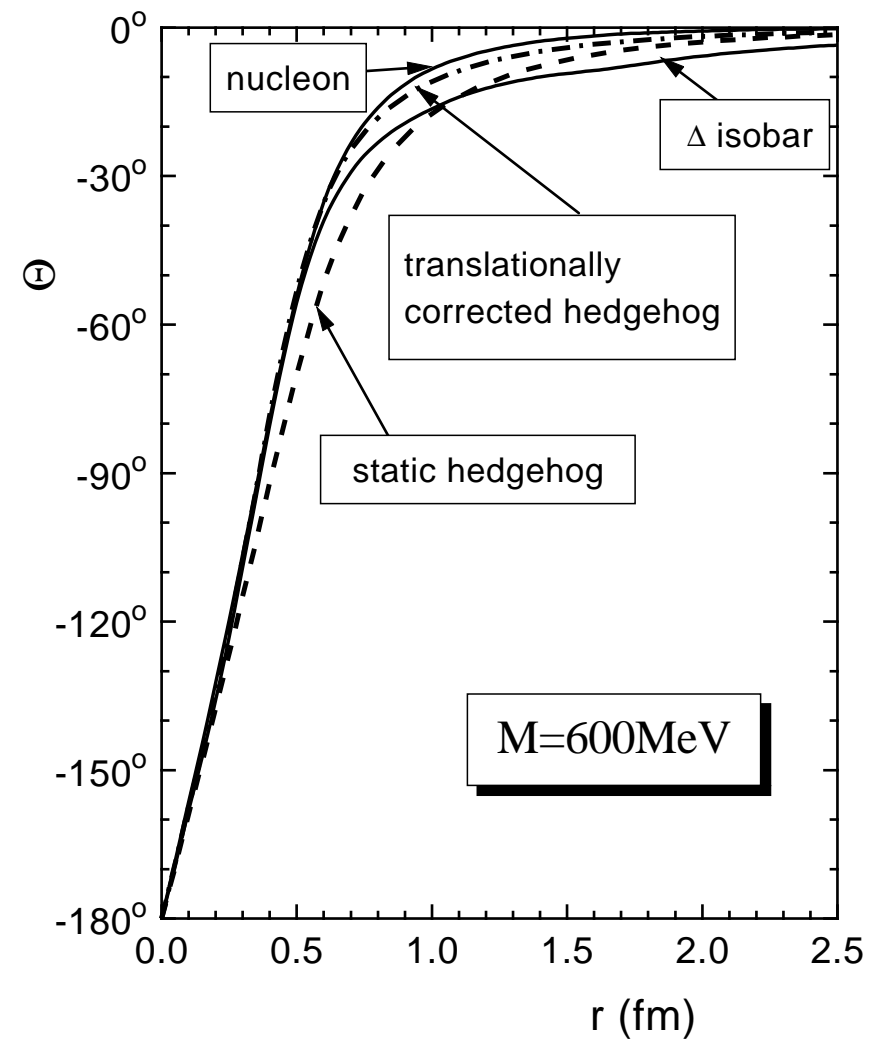

Fig. 4: Profile functions $\Theta(r)$ for the static hedgehog soliton (broken line) in comparison with the corrected profiles for nucleon and $\Delta$ isobar (full lines) calculated for the constituent quark mass $M=$ $600 \mathrm{MeV}$. Additionally the self-consistent profile for a translationally corrected soliton without rotational correction is shown (dashed-dotted line).

Fig. 4 illustrates the general features of the modification in the profile function caused by the correction terms. We selected the relatively large constituent quark mass of $600 \mathrm{MeV}$ in order to get a pronounced effect. Translational corrections modify the behavior of the meson profile at small radii while rotational corrections affect the asymptotics at large radii. The first ones increase the slope of the internal linear part and make the soliton smaller. The size of the mean field and of the corresponding quark distribution is a result of the balance between the diverging Fermi motion of the quarks and the attraction between them. Subtracting the translational zero-point energy the balance is disturbed since the 
Fermi motion is reduced and the attraction dominates. The soliton shrinks until the increasing Fermi motion balances the attraction.

Rotational corrections are different in sign for nucleon and $\Delta$ isobar and manifest themselves at large $r$. They act like a centrifugal force, which is negative for the nucleon and positive for the $\Delta$ isobar. In accordance with eq. (16) and fig. 2 the exponential descent is larger for nucleons and smaller for $\Delta$ isobars. Fig. 4 shows that rotational corrections modifies the meson field already at intermediate distances from the center of the soliton $(r \gtrsim 0.6 \mathrm{fm})$.
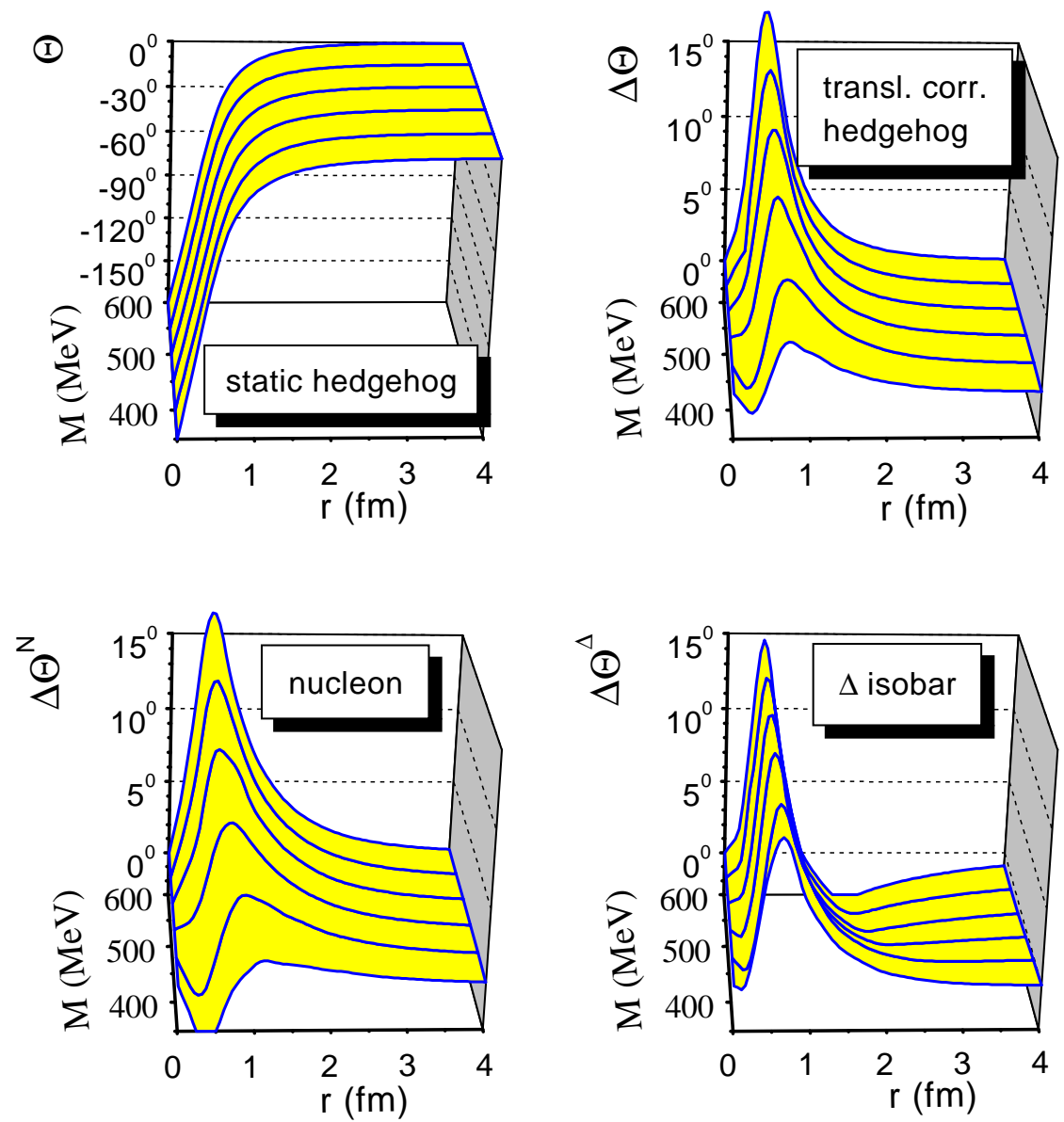

Fig. 5: Upper left figure: Profile function $\Theta(r)$ for the static soliton in the mass region $350 \mathrm{MeV} \leq M \leq 600 \mathrm{MeV}$; Upper right figure: Deviation $\Delta \Theta(r)$ of the translationally corrected profile from the static one; Lower figures: Deviations $\Delta \Theta^{\mathrm{N}, \Delta}(r)$ of the translationally and rotationally corrected meson profiles for nucleon and $\Delta$ isobar, respectively.

Fig. 5 displays the self-consistent meson profiles and the deviations of the corrected profiles from the static ones in the whole mass region. As already 
shown in ref. [3] the shape of the static profile is practically independent of the constituent quark mass (upper left figure). The distortion of the static meson profile by the correction terms turns out to be more significant than its variation in dependence on $M$. The effect of the corrections grows in dependence on the constituent quark mass $M$. Heavier constituent quarks are stronger bound and produce smaller solitons. Their center-of-mass energy is larger, while the moment of inertia is smaller. Both factors increase the correction term and their influence on the meson profile.

\section{Expectation values of the soliton calculated with corrected meson profiles}

It is the aim of this paper to evaluate the influence of translational and rotational corrections of the self-consistent meson fields and to estimate their effect on expectation values of solitonic observables. For that reason we compare expectation values of observables which are calculated with corrected and uncorrected meson profiles, respectively. In the present paper we will not consider pushing and cranking correction to the observables themselves. A full treatment of symmetry restoration would have to include these corrections as well. In several cases they have turned out to be rather important [25, 26] and therefore must be taken care of before the results are compared with experimental data. This will be the subject of a forthcoming analysis.

Generally it can be said that the effect coming from the difference between uncorrected and corrected meson profiles grows with increasing constituent quark mass $M$ in accordance with the increasing size of the correction terms ( $c f$. fig. 1). At small constituent quark masses $M$ the valence quarks give the dominating contribution to the expectation value in most cases. Increasing the quark mass the Dirac sea gets more and more polarized and its contribution gets larger. Covering the mass region $300 \mathrm{MeV} \leq M \leq 600 \mathrm{MeV}$ we are able to control the ratio between the contributions resulting from valence and sea quarks.

Fig. 6 illustrates the energy of the soliton and its components. The total corrected energies for nucleons and $\Delta$ isobars are shown in the upper part. The experimental nucleon mass is reached at $M \approx 350 \mathrm{MeV}$, but the predicted mass of the isobar is remarkably smaller than the experimental value in the whole mass region. Pushing and cranking corrections in the meson field decrease the mass furthermore. The difference between full and broken lines indicates the gain of energy which is obtained if one minimizes the corrected soliton energy instead of its static value. Here we compare the corrected total soliton energy (9) calculated for the corrected profiles $\Theta_{\text {corr }}^{T}$ with the same energy calculated for the static profile $\Theta$. While the total energies do not differ very much from each other valence and sea quark energies are rather different in both cases (central and lower 
part of fig. 6). For the static soliton we have a dominating valence contribution at small constituent quark masses $(M \lesssim 420 \mathrm{MeV})$. In the energy of the corrected soliton, the valence quarks dominate up to larger masses $(M \lesssim 500 \mathrm{MeV})$. The correction terms even prevent the valence quark energy from getting negative what happens at $M \approx 750 \mathrm{MeV}$ in the uncorrected case [3]. The field energy (2) of the mesons is strongly affected by the correction (up to a factor of 2). However, its contribution to the total soliton energy is marginal due to the restriction to the chiral circle.

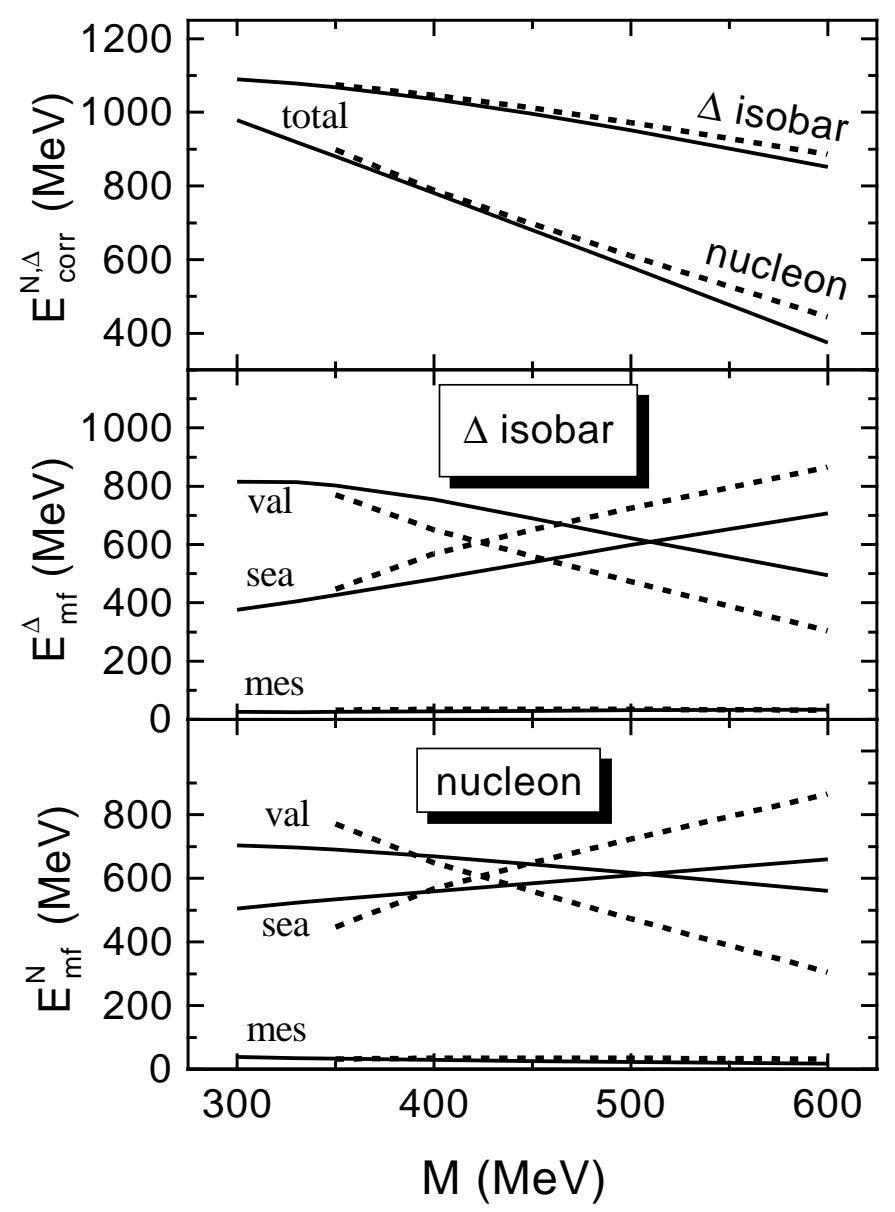

Fig. 6: Upper part: Total corrected energies $E_{\text {corr }}^{\mathrm{N}, \Delta}$ for nucleon and $\Delta$ isobar calculated for the static meson profile $\Theta$ (broken lines) in comparison to the same energy calculated for the corrected profiles $\Theta_{\text {corr }}^{\mathrm{N}, \Delta}$ (full lines).

Central and lower part: Contributions of the valence (val) and sea quarks (sea), and of the mesonic field (mes) to the total soliton energy $E_{\mathrm{mf}}$ calculated for the static meson profile (broken lines) and for the corrected profiles (full lines) as a function of the constituent quark mass M.

The effect of the corrections on an expectation value depends crucially on the relation between valence and sea-quark contributions. If the valence quarks, which are usually well bound inside the soliton, give the dominating contribution, the shrinking of the meson profile in the internal region (see fig. 4) is the most important effect. It is caused by the center-of-mass correction and is independent of the isospin quantum number. Rotational corrections have almost no effect on the valence quarks. Sea quarks are either less bound or unbound and hence experience the action of rotational corrections which are opposite for nucleons and 
$\Delta$ isobars. Considering the sea contribution of an expectation value in dependence on the radius $r$ one notices that contributions from the internal part of the soliton may cancel out contributions from external layers. This is not the case for the valence part since the corresponding wave function has no node. Modifications in the meson profile influence the complicated interplay between positive and negative contributions and may affect the total sea contribution considerably. As a result sign and size of the effect depend strongly on the observable. In the following we give some examples in order to illustrate what might happen.

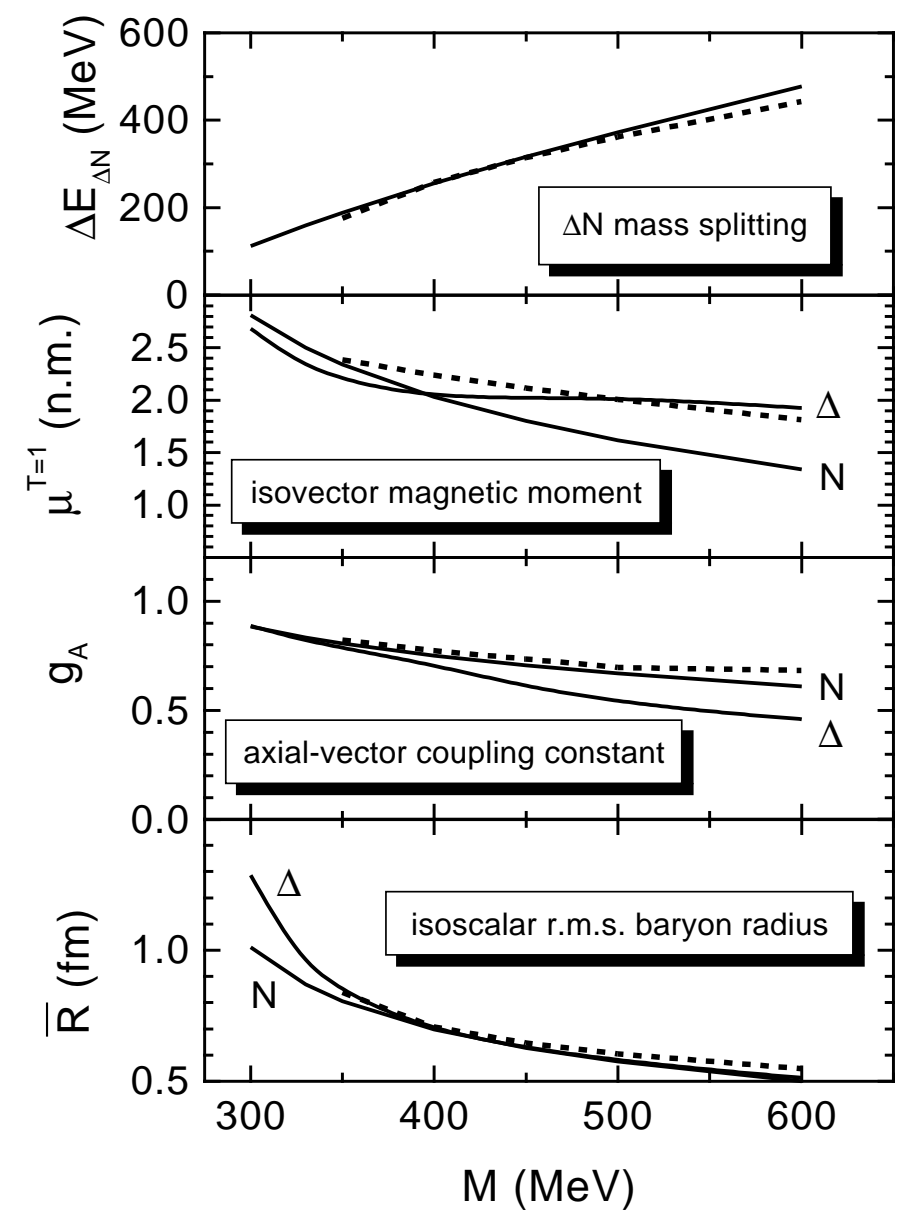

Fig. 7: $\Delta$-nucleon mass splitting $\Delta E_{\Delta \mathrm{N}}$, isovector magnetic moment $\mu^{T=1} \equiv \mu_{\mathrm{p}}-\mu_{\mathrm{n}}$, axialvector coupling strength $g_{\mathrm{A}}$ and isoscalar root mean-squared (r.m.s.) baryon radius $\bar{R}$ calculated for the translationally and rotationally corrected nucleon $(\mathrm{N})$ and $\Delta$ profiles (full lines) in comparison to the same quantities for the static soliton (broken lines), as a function of the constituent quark mass $M$.

The (isoscalar) r. m. s. radius $\bar{R}$ of the quark distribution (lower part of fig. 7 ) is dominated by the valence quarks by nearly 95 percent. Hence nucleon and $\Delta$ isobar have almost the same radius. The reduction of the radius with respect to the static hedgehog by a few percent is in quantitative agreement with the steeper behavior of the corrected meson profile in the central region of the soliton ( $c f$. figs. 4,5$)$. For very small constituent quark masses the valence quarks are so weakly bound that they may reach larger separations from the center. Here they are exposed to the rotational corrections which lead to a considerable shrinking for 
the nucleon and a remarkable swelling for the $\Delta$ isobar with respect to the static hedgehog. The r. m. s. radius is particularly sensitive to the quark distribution at large radii since the quark density is weighted by a factor $r^{4}$.

Now let us investigate the axial-vector coupling constant $g_{\mathrm{A}}$ of the nucleon (fig. 7). In order to study the pure influence of the corrected meson field we consider the expectation value of the same operator [3] without any rotational corrections. This uncorrected observable accounts for roughly 65 percent of the experimental value of $g_{\mathrm{A}}[25,26]$. Apart from rotational corrections the coupling constant for the $\Delta$ isobar differs from the nucleon by an additional factor which results from the different spin-isospin structure of both particles. The figure shows the $g_{\mathrm{A}}$ of the proton calculated for meson profiles which have been corrected for both nucleons and $\Delta$ isobars. Here the dominance of the valence quarks is less pronounced as in the case of $\bar{R}$ and the different asymptotic behavior of nucleon and $\Delta$ profiles results in slightly different expectation values. Roughly spoken translational and rotational corrections cancel each other for the nucleon while they add up (negatively) for the $\Delta$ isobar.

In the isovector magnetic moment of the nucleon $\left(\mu_{\mathrm{N}}^{T=1} \equiv \mu_{\mathrm{p}}-\mu_{\mathrm{n}}\right)$, contributions from larger radii are favored by a factor $r^{3}$ [1, 26]. Sea contributions amount to $20 \ldots 30$ percent of the total value. This leads to a noticeable difference between the expectation values for nucleon and $\Delta$ profiles. Here, the correction is negative for the nucleon and almost cancels out for the $\Delta$ isobar.

In the last example we consider the delta-nucleon mass splitting $\Delta E_{\Delta \mathrm{N}}$. In the case of the static soliton the splitting results solely from the different cranking energies of both particles and is uniquely determined by the moment of inertia $I$

$$
\Delta E_{\Delta \mathrm{N}}=\frac{3}{2 I} \text {. }
$$

Using the corrected meson profiles, which are slightly different for nucleons and $\Delta$ isobars, the self-consistent values of static energy (1), translational zero-point energy (10) and of the moment of inertia are different as well. Now the mass difference is given by

$$
\Delta E_{\Delta \mathrm{N}}^{\mathrm{corr}}=\left[E_{\mathrm{mf}}^{\Delta}-E_{\mathrm{mf}}^{\mathrm{N}}\right]-\left[E_{\text {trans }}^{o, \Delta}-E_{\text {trans }}^{o, \mathrm{~N}}\right]+\frac{3}{4}\left[\frac{1}{I^{\Delta}}+\frac{1}{I^{\mathrm{N}}}\right]
$$

where the additional upper index $N, \Delta$ indicates the corresponding particle. In the upper part of fig. 7 we see that the simple expression (18) with the static moment of inertia agrees with the more complicated expression (19) for corrected profiles very well. The analysis of the various terms in eq. (19) shows that the first two differences are rather small $(\approx 10 \mathrm{MeV})$. In spite of slightly different moments ( $c f$. fig. 2) of nucleon and $\Delta$ isobar the last term is nearly identical with the result of eq. (18). That means that the harmonic average defined by

$$
\frac{1}{I^{\mathrm{av}}}=\frac{1}{2}\left[\frac{1}{I^{\Delta}}+\frac{1}{I^{\mathrm{N}}}\right]
$$


agrees with the moment of the static hedgehog soliton ( $c f$.fig. 2). The mass splitting provides an example of an almost complete canceling of meson-field corrections.

The magnitude of the evaluated effects does not exceed a level which describes the reliability of the model in particular in the physically relevant region of small constituent quark masses. The situation may be different if one considers observables with oscillating radial dependence, e.g. formfactors. Here a minor modification in the meson profile may have a larger effect.

\section{Conclusions}

We have evaluated pushing and cranking corrections on solitonic meson fields of the bosonized NJL model in one-loop approximation and studied their effect on the quark distribution and other observables of the soliton. The corrected fields have been obtained by minimizing an energy functional which differs from the mean-field energy by several correction terms. These terms have been introduced to remove the energy of spurious translational and rotational modes and to equip the soliton with the quantum numbers of nucleon or $\Delta$ isobar on a semi-classical level. The meson fields are restricted to the chiral circle and to hedgehog configurations with a modified asymptotic behavior.

We have studied solitons and their expectation values in the region $300 \mathrm{MeV} \leq$ $M \leq 600 \mathrm{MeV}$ of the constituent quark masses $M$. The results illustrate the response of the meson field to the corrections and quantify their effect on expectation values. Despite the big correction terms (up to 50 percent of the total soliton energy) meson and quark fields are only moderately affected. The (approximate) restoration of translational symmetry increases the slope of the meson profile in the central region and makes the soliton smaller. This effect prevents the valence quarks from diving into the negative-energy region up to very large constituent quark masses. It stabilizes the soliton by reducing the kinetic energy of the quarks. As a result, a stable soliton exists at quark masses below $350 \mathrm{MeV}$ (up to $\approx 300 \mathrm{MeV}$ ) while the uncorrected soliton is heavier than 3 free quarks and de-

cays, in this mass region. Rotational corrections affect the asymptotic behavior of the fields at large radii. They depend on the spin and isospin quantum numbers and therefore have different effects on nucleon and $\Delta$ isobar. Both translational and rotational corrections grow with increasing constituent quark mass.

Expectation values of the soliton are affected by the corrections to various extents. The isoscalar r.m.s. radius of the nucleon is reduced by only a few percent $(\approx 3-5$ percent $)$. Larger changes can be observed if one considers valence and sea quark contributions separately. Axial-vector coupling strength and isovector magnetic moment are modified up to 40 percent. In the physically relevant region of small constituent quark masses $(350 \mathrm{MeV} \lesssim M \lesssim 450 \mathrm{MeV})$, the corrections do not exceed 20 percent. The $\Delta$-nucleon mass splitting is practically not affected 
by the corrections despite the slightly different moments of inertia calculated for nucleon and $\Delta$ isobar.

The experimental values of $\mathrm{r}$. m.s. radius and $\Delta \mathrm{N}$ mass splitting are well reproduced for light constituent quark masses around $400 \mathrm{MeV}$. Here, the effect of translational and rotational corrections to the mean field is small and does not exceed the level of accuracy of the model and its numerical treatment. The quantities $g_{\mathrm{A}}$ and $\mu^{T=1}$ are stronger affected by the corrections. Up to now the theoretical values underestimate the experimental ones significantly. The calculated values are even smaller with the corrections than without. However it must be noted that our treatment is incomplete, because in order to be able to compare with the experiment one has to take into account that there are also rotational corrections to the corresponding operators, which have turned out be quite large [1].

\section{ACKNOWLEDGEMENT}

The authors wish to acknowledge stimulating discussions with K. Goeke, H. Reinhardt, R. Alkofer, H. Weigel, J. Berger and Chr. V. Christov. The paper was supported by the Bundesministerium für Forschung und Technologie (contract 06 DR 666).

\section{References}

[1] C.V. Christov, A. Blotz, H.-C. Kim, P.V. Pobylitsa, T. Watabe, T. Meissner, E. Ruiz Arriola and K. Goeke, Prog. Part. Nucl. Phys. 37, 1 (1996).

[2] R. Alkofer, H. Reinhardt and H. Weigel, Phys. Rep. 265, 139 (1996).

[3] R. Wünsch, K. Goeke and T. Meissner, Z. Phys. A348, 111 (1994).

[4] R.E. Peierls and J. Yoccoz, Proc. Phys. Soc. 70, 381 (1957).

[5] R.E. Peierls and D.J.. Thouless, Nucl. Phys. 38, 154 (1962).

[6] B. Moussalam and D. Kalafatis, Phys. Lett. 272B, 196 (1991).

[7] G. Holzwarth, Phys. Lett. 291B, 218 (1992).

[8] R. Rajaraman, Solitons and Instantons (North-Holland, Amsterdam, 1982).

[9] H. Weigel, R. Alkofer and H. Reinhardt, Nucl. Phys. A582, 484 (1994).

[10] P. Ring and P. Schuck, The Nuclear Many-Body Problem (Springer, Heidelberg, 1980).

[11] H. Reinhardt and Wünsch, Phys. Lett. 215B, 577 (1988). 
[12] G. S. Adkins, C. R. Nappi and E. Witten, Nucl. Phys. B228, 552 (1983).

[13] P.V. Pobylitsa, E. Ruiz Arriola, T. Meissner, F. Grümmer, K. Goeke and W. Broniowski, J. Phys. G18, 1455 (1992).

[14] J. Schwinger, Phys. Rev. 82, 664 (1951).

[15] H. Reinhardt, Nucl. Phys. A503, 825 (1989).

[16] H. Reinhardt and R. Wünsch, Phys. Lett. B230, 93 (1989).

[17] T. Meissner and K. Goeke, Nucl. Phys. A524, 719 (1991).

[18] A. Kamlah, Z. Phys. 216, 52 (1968).

[19] M.C. Birse, Prog. Part. Nucl. Phys. 26, 1 (1990).

[20] J.-P. Blaizot and G. Ripka, Phys. Rev. D38, 1556 (1988).

[21] U. Post and U. Mosel, Nucl. Phys. A499, 789 (1989).

[22] N. Dorey, J. Hughes and M.P. Mattis, Phys. Rev. D50, 5816 (1994).

[23] S. Kahana and G. Ripka Nucl. Phys. A419, 462 (1984).

[24] M. Schleif and R. Wünsch, Research Center Rossendorf, Report No. FRZ-55, 1994 (unpublished).

[25] M. Wakamatsu and T. Watabe, Phys. Lett. 312B, 184 (1993).

[26] C.V. Christov, A. Blotz, K. Goeke, P. Pobylitsa, V. Petrov, M. Wakamatsu and T. Watabe, Phys. Lett. 325B, 467 (1994). 